\title{
Violence and aggression at a substance misuse treatment clinic in Ireland
}

\author{
Chinedu J. Iro, ${ }^{1}$ Nnamdi Nkire, ${ }^{2}$ John $\mathrm{O}^{\prime}$ Connor $^{1}$
}

The Psychiatrist (2011), 35, 216-219, doi: 10.1192/pb.bp.110.031468

${ }^{1}$ Drug Treatment Centre Board, Dublin ${ }^{2}$ Cavan and Monaghan Mental Health Service, Ireland

Correspondence to Chinedu J. Iro (ciro@dtcb.ie)

First received 7 Jun 2010, final revision 12 Nov 2010, accepted 23 Nov 2010

\begin{abstract}
Aims and method To report the rates of violent and aggressive incidents at a drug treatment clinic using a newly introduced incident reporting tool (STARSWeb) and to describe the management strategies currently employed in the management of incidents at the centre. This involved the review of all completed incident reports for the year 2008 and the examination of relevant patient factors.
\end{abstract}

Results There were 276 documented incidents at the centre in 2008 . The majority of incidents (72.4\%) involved verbal abuse and threatening behaviours. Males were responsible for the majority of incidents. Two-thirds of the clients' urine samples were positive for illicit substances at the time of the incidents.

Clinical implications Violent and aggressive incidents in healthcare settings continue to pose a real challenge to both service providers and service users. An accurate system of reporting of such incidents is indispensable in guiding policy development and management strategies. The STARSWeb system offers a significant improvement in incident reporting towards the attainment of these goals.

Declaration of interest None.
It is well recognised that working in healthcare settings is associated with significant occupational hazards. These may take the form of accidental injury, exposure to noxious or infective agents and direct violence against staff and/or patients. Direct violence and threats to healthcare staff may exert adverse effects on their psychological, emotional and social well-being, with resultant decline of morale and reduction in overall productivity of the organisation. ${ }^{1}$ Studies which have examined this phenomenon have focused on general hospital settings, general practice and the emergency departments, ${ }^{2-4}$ and specific patient groups such as psychiatric in-patients ${ }^{2}$ or those with particular diagnoses, for example schizophrenia.

The Drug Treatment Centre Board is the foremost tertiary centre for the treatment of opiate dependence in Ireland. The centre deals with complex cases of opiate dependence by providing prevention, treatment, rehabilitation and after-care programmes for patients. It is a consultant-led service with well-resourced multidisciplinary teams. Pathways to care include self-referrals as well as referrals from general practitioners (GPs), psychiatrists, general hospital doctors and the prison service.

As common to all healthcare settings, staff at the centre are exposed to violent and aggressive incidents. Although the prevalence of violence and aggression at the centre has been well studied, there is a paucity of literature on the general management of violent and other incidents in addiction treatment settings. There have been two previously published studies of violence and aggression in the centre., ${ }^{1,5}$ These studies used similar methods and revealed the trends over time.

The primary means of assessing violence at the centre is a review of incident reports. In mid-2007, the centre changed its method of incident reporting to a more comprehensive clinical incident reporting system known as the STARSWeb. ${ }^{6}$ This is a well-structured, validated and reliable tool, which has wide acceptance. It was first introduced in Ireland in 2003 and was to serve as a secure, web-based IT system linking hospitals and other healthcare enterprises to the clinical indemnity scheme core database. ${ }^{7}$ The STARSWeb system has the advantage of improved accuracy of incident reporting and enables healthcare organisations to react quicker in taking corrective measures. ${ }^{6}$

We sought to analyse reported incidents of violence and aggression in the centre since the introduction of the STARSWeb system, examining the crude rates, demographic patterns and other associated factors.

\section{Method}

We retrospectively reviewed all reported incidents at the centre for the year 2008, recorded on the STARSWeb incident report forms. We also obtained additional information from the electronic patient records of patients identified from the incident report forms. Data collected included baseline demographic details, details of the incidents, forensic history, history of violence, stability of 
drug treatment (taken as presence of at least one positive opiate sample in a 2-week period preceding an incident), location of incident, history of mental illness and the context of the incident.

Previous studies examining incidents of violence and aggression at the centre adopted a hierarchical system in which only one incident (the most significant) was included in the analysis, thereby discounting other 'minor' associated incidents. This meant that there was an under-recording of the actual number of incidents. The identification of individual incidents is important as this gives a more representative picture of the extent of violence and aggression and provides a better guide for managing such incidents. An illustration of this approach is the case of a patient who was verbally abusive to the nursing staff, damaged property within the waiting area and directly threatened violence to the security staff. Although this was recorded on one form (one episode), it actually comprised three distinct incidents (verbal abuse, property damage and threatening/aggressive behaviour).

The study was approved by the local research ethics committee. Incidents were individually identified and classified into seven groups (Table 1), rather than three categories used in previous studies. ${ }^{1,5}$ Data were codified and analysed with SPSS version 15 for Windows.

\section{Results}

During the study period (2008), a total of 771 individuals attended the service and there were a total of 276 documented incidents involving 156 episodes (12 episodes of occupational injuries were excluded from this analysis). Overall, 98 patients were involved (12.7\%), equivalent to 202 per 1000 patients/year and with a rate of 358 incidents per 1000 patients/year (1.8 incidents per episode). This is much higher than the previous figure of 85 per 1000 patients/year reported by Whitty \& O'Connor. ${ }^{1}$

Thirty-two patients had offended repeatedly, with five individuals being involved in more than three incidents each. One person was responsible for 12 incidents (4.4\%).

The percentage of patients involved in incidents per year was higher in 2008 compared with the previous study ${ }^{1}$ $(12.7 \%$ v. 8.4\%). There was also an increased rate of recidivism within the study period $(32.7 \%)$ compared with a previously reported figure $(10 \%) .^{5}$ Two-thirds of individuals involved in such incidents $(72 \%)$ also had a history of violence or aggressive behaviour in the centre. The mean age of patients with offending behaviour was slightly lower than the centre's average (33v. 36 years).

Males were responsible for $79 \%$ of incidents but were less likely to be involved in physical violence (odds ratio $\mathrm{OR}=0.57$ ). This was likely due to the fact that fighting among patients was more common with females. There was no statistically significant gender difference in the overall propensity to violence.

Physical violence directed against staff reduced by $60 \%$ since the last review (Table 1). This might be a reflection of the effectiveness of security measures put in place to protect the front-line staff.

Whereas no cases of racial abuse were reported in $2000,{ }^{5}$ by 2003 the figure was $11 \%$ of all reported incidents. ${ }^{1}$
This is likely a reflection of the changing staff ethnic profile which occurred around that time. In our study, the rate of racial abuse was $6.5 \%$ (a reduction of $4.6 \%$ ).

During the reference period, two-thirds of patients had opiate-positive urine samples in the 2 weeks preceding an incident. Less than half (40\%) had a psychiatric history; psychiatric comorbidity did not appear to be a factor as the prevalence of dual diagnosis in the centre is $43 \%{ }^{8}$

Two-thirds of incidents (64.9\%) occurred during the morning session. This might have been due to the absence of individualised appointments for attendance, with the result that the bulk of patients presented during the morning session.

A positive finding was the fact that there were very few incidents occurring in confined areas such as the consulting rooms and treatment rooms (Table 2). This might suggest that clinical risk assessment methods were applied and that staff were more alert and more likely to deal with patients with perceived high risk in open and supported areas.

Table 1 is a comparative analysis of those subject to aggressive and/or violent behaviour between a previous study $^{1}$ and ours. Whereas the rates of violence against

\begin{tabular}{|c|c|c|c|c|}
\hline \multicolumn{5}{|c|}{$\begin{array}{l}\text { Table } 1 \text { Incidents of verbal abuse and physical violence } \\
\text { in the centre compared with a previous audit } \\
\text { by Whitty \& } O^{\prime} C^{\prime} \text { onnor }\end{array}$} \\
\hline \multirow[b]{2}{*}{ Staff affected } & \multicolumn{2}{|c|}{ Verbal abuse, $n$} & \multicolumn{2}{|c|}{ Physical assault, $n$} \\
\hline & $\begin{array}{c}2002+ \\
2003\end{array}$ & 2008 & $\begin{array}{c}2002+ \\
2003\end{array}$ & 2008 \\
\hline Nurse & 68 & 55 & 2 & 2 \\
\hline Doctor & 49 & 31 & 3 & 1 \\
\hline General assistant & 60 & 21 & 8 & 1 \\
\hline Security staff & 26 & 10 & 6 & 0 \\
\hline Other staff & 7 & 3 & 1 & 0 \\
\hline Other patient & 11 & 4 & 13 & 5 \\
\hline Total & 221 & 124 & 33 & 9 \\
\hline
\end{tabular}

Table 2 Location and frequency of incidents at the centre in 2008

\begin{tabular}{lc} 
Location & $n(\%)$ \\
\hline General waiting area (floor) & $54(30)$ \\
\hline Dispensing area (hatch) & $44(24)$ \\
\hline Doctor's interview room & $2(1)$ \\
\hline Treatment room & $3(2)$ \\
\hline Outside the building & $8(4)$ \\
\hline Toilet & $10(6)$ \\
\hline Security search area & $13(7)$ \\
\hline Amicus (safe area) & $23(12)$ \\
\hline Office/corridor & $9(5)$ \\
\hline Other $^{\text {a }}$ & $18(10)$ \\
\hline Total $^{2}$ & $184(100)$ \\
\hline
\end{tabular}

a. Includes incidents occurring in the lift, over the telephone and in the crèche. 
nurses and doctors increased in 2008, the opposite was true for other staff categories.

Table 2 lists the locations in which the incidents occurred. The bulk of incidents happened in the waiting area followed by the nurses' dispensing area.

Table 3 shows the actions taken following a review of the incidents by the clinical team. The most common course of action was the transfer of the patient involved to a time+restricted section of the centre (Amicus).

Fig. 1 is a graphical representation of the specific types of incidents.

\section{Discussion}

Violence in healthcare settings is a real and challenging problem that seems to be escalating. There were 6500

\begin{tabular}{|lr|}
\hline Table 3 & $\begin{array}{l}\text { Actions taken following review of incidents } \\
(n=175)^{\mathrm{a}}\end{array}$ \\
\hline Actions taken & $n(\%)$ \\
\hline Amicus (safe area) & $68(39)$ \\
\hline Warned & $28(16)$ \\
\hline Lost takeaway methadone & $9(5)$ \\
\hline Suspended & $8(5)$ \\
\hline Review of dose of methadone & $6(3)$ \\
\hline Psychiatric review & $5(3)$ \\
\hline Referral to external hospital & $5(3)$ \\
\hline Time sanction & $4(2)$ \\
\hline Low dose methadone instituted & $3(2)$ \\
\hline Police involvement & $3(2)$ \\
\hline Review of procedure & $2(1)$ \\
\hline No action & $34(20)$ \\
\hline
\end{tabular}

a. Some incidents involved more than one action.

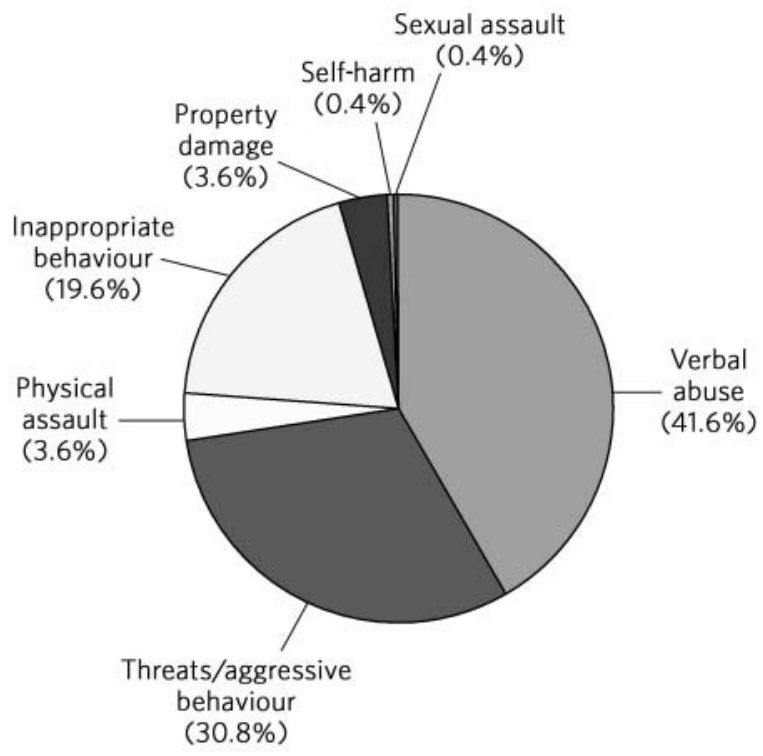

Fig 1 Type of incident. violent incidents against National Health Service (NHS) trust staff in $1999 .^{9}$ Rates of violence were higher in mental health trusts and towards nurses. ${ }^{10}$ It is also believed that there are significant levels of underreporting. The national audit office reported incremental rates of $13-20 \%$ during the period 1998-2002. ${ }^{11}$ The management of violence in healthcare settings is associated with specific challenges which vary from one setting to the next. These include physical strategies, security apparatus, medication and risk forecast. It is generally accepted that no forms of violence towards healthcare staff should be tolerated, an approach that was adopted in the NHS 'zero tolerance' campaign.'

In 1998, the Royal College of Psychiatrists issued guidelines for the management of imminent violence in clinical settings. ${ }^{12}$ This recognised the multifactorial genesis of violence including patient, environmental and interpersonal factors. It similarly advocated a multifactorial approach to violence management including governance structures, modification of the physical environment, the development of protocols, staff training, risk prediction, time out, use of medication and prompt addressing of all incidents of violence. Many of these recommendations do not, however, apply to addiction clinics, for example the use of time out or seclusion as practised in psychiatric settings.

Concomitant with the provision of addiction treatment, the Drug Treatment Centre Board has a duty to actively support its employees within its relatively high-risk environment. The formulation of protocols for managing incidents in the centre is dependent on the formal reporting of all incidents as this provides the statistical and qualitative information necessary for guiding resource provision towards addressing violent incidents. The introduction of the STARSWeb system was intended to improve incident reporting.

\section{Behavioural management strategies}

Several strategies are employed in managing behaviours within addiction treatment settings including the Drug Treatment Centre Board in Dublin. Preventative environmental measures include the use of metal detectors and scanners, the design of the waiting areas, the use of a numbered queuing system to avoid clustering at the dispensing areas and CCTV. All furniture within waiting areas and consulting rooms is fixed. Other environmental measures include active management of loitering (which may indicate drug dealing) via security patrols around the premises.

Clinical/operational measures include education and training of staff in dealing with individuals on methadone treatment. All new staff members undergo a compulsory induction programme which incorporates breakaway training (non-violent crisis intervention training). Staff members are equipped to develop skills in the recognition of high-risk situations, with referral of such individuals to a safe section of the clinic (Amicus). In cases where patients are known to be feuding, they are given appointments to attend at different times of the day.

All incidents are followed by a multidisciplinary team review. This process considers the incident from a multifactorial perspective and applies interventions which are specific to a person and aimed to ultimately benefit 
them while protecting the smooth operation of the centre. In addition, the centre has a quarterly security review.

Contingency management which is based on operant conditioning is commonly utilised in addiction settings. Although associated with controversy, it has been found to be very effective in individuals on methadone treatment. ${ }^{13}$ Desirable behaviours are encouraged through rewards such as granting of takeaway methadone and other privileges. Similarly, undesirable or inappropriate behaviours are dealt with through the withdrawal of privileges, the application of time restrictions and in extreme cases suspension of patients from the clinic. There are obvious ethical considerations involved in application of any sanctions if these are not to be viewed as punishment. Ideally, these measures should not have a significant negative impact on the core treatment being provided. These considerations, however, limit the effectiveness of such strategies. In our study a small number of individuals $(<10 \%)$ were suspended or had their methadone dose reviewed, while only three individuals were formally charged by the police.

There is also the possibility of acclimatisation to sanctions, which sees the effectiveness of any interventions reduce with time.

Specific strategies in use for managing incidents at the Drug Treatment Centre Board are detailed in Table 3 and range from verbal warnings, through sanctions such as loss of takeaways, to security reviews and referral to the police. In addition, all patients are required to sign a behaviour contract before being admitted to the centre. These measures have had varying degrees of success as evidenced by our results. The 'zero tolerance policy' approach to racial abuse adopted by the centre following the emergence of such behaviour in a previous study ${ }^{5}$ appears to have resulted in the reduction seen in our study. This approach involved educational campaigns in the centre. In the event of racial abuse, there was a protocol in place for a team to review the patient before the next dispensing of methadone, with the opportunity for an apology or the application of stipulated sanctions when indicated.

Contrary to the previous study, ${ }^{5}$ the requirement to sign a written 'behaviour contract' had not been found to be effective in reducing violent and aggressive incidents.

The reasons for the increased rates of reported incidents of violence and aggression in our study are unclear. It is possible that better reporting with STARSWeb contributed to this apparent increase.

\section{Clinical implications}

The introduction of the STARSWeb incident reporting system has been a success in that it has resulted in more detailed reporting of violent and aggressive incidents. This has meant that individual episodes are now reviewed in terms of the specific incidents involved. The security measures put in place following the recommendations of previous studies have been successful to a large extent in reducing the total number of incidents of physical violence and racial abuse. However, in view of the relatively large number of reported incidents, further steps need to be taken.

It would be important to re-audit violent and aggressive incidents at the centre to examine trends since the introduction of the STARSWeb system as this would give a clearer picture as to the trend of incidents over time and provide more information on effective interventions. There needs to be ongoing training of all new staff including rotating doctors on the use of the STARSWeb as well as refresher courses for existing staff. Practical measures to improve the management of incidents might include a consideration of patient scheduling to avoid overcrowding at particular times of the day. Other strategies would need to be explored and would need to comprise a combination of addiction, psychological, behavioural and legal approaches especially to address the highlighted issue of recidivism.

\section{About the authors}

Chinedu J. Iro is a senior registrar in general psychiatry and currently working at the Drug Treatment Centre Board, Nnamdi Nkire is a psychiatry research fellow, St Davnet's Hospital, Cavan and Monaghan Mental Health Service, Monaghan, John O'Connor is a consultant psychiatrist in substance misuse and clinical director, Drug Treatment Centre Board, Dublin, Ireland.

\section{References}

1 Whitty P, O'Connor J. Violence and aggression in the Drug Treatment Centre Board. Ir J Psych Med 2006; 23: 89-91.

2 Omérov $M$, Edman G, Wistedt B. Incidents of violence in psychiatric inpatient care. Nord J Psychiatr 2002; 56: 207-13.

3 Baker L, Murray G, Bell P, Sutton A, Dean S. Rural general practitioner experience of work-related violence in Australia. Austr J Rural Health 2003; 11: 231-6.

4 Ryan D, Maguire J. Aggression and violence - a problem in Irish accident and emergency departments. J Nurs Management 2006; 14: 106-15.

5 Fahy S, Brown R, Sloan D, Keating S, O'Connor J. An audit of violent incidents in the drug treatment center. Ir J Psych Med 2000; 17: 29-33.

6 State Claims Agency. Clinical Incident Reporting System - STARSWeb. State Claims Agency (http://www.stateclaims.ie/Clinicallndemnity Scheme/introduction.html).

7 Toolkit of Documentation to Support the Health Services Executive Incident Management. Office of Quality and Risk, March 2009.

8 Iro C, O'Connor J. Dual diagnosis in a Dublin tertiary addiction centre a cross-sectional study. Ir J Psych Med 2009; 26: 191-3.

9 Department of Health. We Don't Have to Take This. NHS Zero Tolerance Zone Campaign. TSO (The Stationery Office), 1999.

10 Department of Health. Working Together: Managing Violence, Accidents and Sickness Absence in the NHS. Health Service Circular 1999/229. TSO (The Stationery Office), 1999.

11 National Audit Office. A Safer Place to Work: Protecting NHS from Violence and Aggression. TSO (The Stationery Office), 2003

12 Royal College of Psychiatrists. Management of Imminent Violence: Clinical Practice Guidelines. Royal College of Psychiatrists, 1998.

13 Prendergast M, Podus D, Finney J, Greenwell L, Roll J. Contingency management for treatment of substance use disorders: a meta-analysis. Addiction 2006; 101: 1546-60. 